\title{
Research and Modeling of Nonlinear Acoustic Processes in a Layered Nonlinear Medium with a Porous Fluid-Saturated Inclusion of a Hierarchical Type
}

\author{
Olga Hachay', Veniamin Dryagin², Andrey Khachay ${ }^{3}$ \\ ${ }^{1}$ Institute of Geophysics, Ural Branch of Russian Academy of Sciences, Yekaterinburg, Russia \\ ${ }^{2}$ LLC “NPF Intensonik”, Yekaterinburg, Russia \\ ${ }^{3}$ Ural Federal University, Yekaterinburg, Russia \\ Email: olgakhachay@yandex.ru,vvdryagin@mail.ru, khachay@yandex.ru
}

How to cite this paper: Hachay, O., Dryagin, V. and Khachay, A. (2019) Research and Modeling of Nonlinear Acoustic Processes in a Layered Nonlinear Medium with a Porous Fluid-Saturated Inclusion of a Hierarchical Type. Open Journal of Geology, 9, 497-506.

https://doi.org/10.4236/ojg.2019.99032

Received: August 2, 2019

Accepted: August 31, 2019

Published: September 3, 2019

Copyright ( 2019 by author(s) and Scientific Research Publishing Inc.

This work is licensed under the Creative Commons Attribution-NonCommercial International License (CC BY-NC 4.0).

http://creativecommons.org/licenses/by-nc/4.0/

\begin{abstract}
Problem statement: The results of the study of seism acoustic emission arising in a porous two-phase geological environment under acoustic influence are presented. Acoustic emission arising in reservoirs of oil fields using good observations is considered. The regularity of the emission processes of acoustic emission, which manifests itself in the form of discrete spectra of signals similar to oscillations of nonlinearly coupled oscillators, is shown. Spectra have special characteristics for each type of rock. Applied method and design: An algorithm for modeling the process of resonant acoustic response of a porous fluid-saturated reservoir with hierarchical structure and plastic properties on acoustic frequency excitation is developed. That algorithm is developed as an iterative process for the solution integral and integral-differential equations. The frequencies that are parameters of the direct problem are used from the spectra of observed data of acoustic emission in the oil wells. Typical results: For the first time, it had been found the relation between resonant frequencies of the acoustic emission and plastic properties, these values of frequencies had been used in the algorithm of modeling distribution of longitudinal waves in the fluid saturated nonlinear plastic environment. Concluding note (Practical value/implications): The analysis of these emission processes can serve as a source of information about the filtration-capacitive properties of productive reservoirs of a porous type with a hierarchical structure. It is used by practical data of oil fields of Western Siberia.
\end{abstract}

\section{Keywords}

Seismic Emission after Acoustic Impact, Reservoir of Hierarchical Structure 
with Plastic Properties, Algorithm of 2D Modeling Connected with Energy Spectrograms

\section{Introduction}

Using the method of Acoustic emission (AE) research, it is possible to obtain information about the processes occurring inside the substance of the reservoir in the well. It has an important advantage over other geophysical methods due to its high resolution in the frequency range. For understanding processes in a saturated porous medium and building adequate models, it is not enough to take into account only the linear relationship between the $\mathrm{AE}$ and dynamic processes in the medium, which is a nonlinear system. The principal feature of a nonlinear medium is the interaction of a limited number of waves associated with the conditions of frequency resonance and wave vectors [1]. The study of acoustic emission in samples of sandstones of terrigenous reservoirs under the action of mechanical stresses was carried out at the UIK-AE core research unit [2]. The energy of the acoustic emission signal, which is released in the process of acoustic impact, was determined by the method of calculating the spectral energy density in the whole recorded frequency range from units of hertz to $20 \mathrm{kHz}$ by the Intel Graph program. In accordance with the theory of nonlinear systems, when nonlinearly coupled oscillators interact, processes are possible when the system generates oscillations with combinational frequencies. In this case, the condition of resonance of frequencies is fulfilled and it is indicated that energy can be exchanged between oscillators, in which the energy of the excited high-frequency oscillator is transferred to two low-frequency oscillators or the reverse process, the merging of low-frequency oscillations. Such an interaction has, for example, a place in the system of three nonlinearly coupled oscillators described by a system of equations [1].

$$
\ddot{x}_{i}+\omega_{i}^{2} x_{i}=\mu \alpha_{i} x_{j} x_{k} ; i=1,2,3 ; j, k \neq i
$$

For a small value of $\mu$, this is a weekly nonlinear system. Its behavior is close to the superposition of quasiharmonic oscillations of oscillators with slowly varying amplitudes. Due to the nonlinear coupling, oscillations of two oscillators with frequencies $\omega_{1}$ and $\omega_{2}$ generate oscillations with a combination of frequencies $\omega_{1}$ and $\omega_{2}$ in the system. The action of a small nonlinearity accumulates if the frequency resonance condition is fulfilled:

$$
\omega_{1}+\omega_{2}=\omega_{3}
$$

When sandstone is deformed, which is an inhomogeneous medium characteristic of the oil reservoir, and when an acoustic field is applied, the formation of wave processes takes place in it. The resulting acoustic emission is represented by both a discrete, pulsed, and continuous noise-like component [3] [4]. 


\section{Nonlinearity of Caused Acoustic Emission Investigation in the Oil Well}

The field of acoustic emission and reception of it signals in a wide frequency range is produced by devices located in a single borehole geophysical device that can move along the wellbore during well testing with a given equipment operation algorithm [2]. The equipment operation algorithm consists of the sequential execution of operations for recording the micro-seismic background in the well, acoustic impact on the mountain massif and re-registration of the signals of emission immediately after the impact. The energy of the induced acoustic emission, which is released in the process of acoustic impact, was determined by the method of calculating the spectral energy density similarly to the method of core research using the Intel Graph 2 program. Figure 1 shows an example of an acoustic emission signal in the BS10 reservoir of a terrigenous type of the Tevlin-Russkinskoye field in Western Siberia during an acoustic impact. On average, the increase in the SAE signal after the acoustic impact in the reservoir saturated with oil is several tens of percent relative to the background level. At the same time, individual acoustic emission events in the form of single actions of emission sources occur randomly and have characteristic parameters of the signal pulses of a finite duration of a certain form. At the same time, the presence of discrete frequencies with a certain maximum energy value is clearly manifested. Spectral analysis of the acoustic emission signal with a duration of one second (Figure 2) is performed using a window Fourier transform, in a sliding window of a given length. The transformation parameters were selected in an optimal way to identify the details of the process in time, which are characteristic of this type of reservoir. The spectrogram shows the distinct discrete nature of the set of frequencies in the signal caused by acoustic emission. At the same time, there is an increase in the energy of acoustic emission at discrete frequencies by several times as compared with the micro-seismic background. In this spectrum, you can select some discrete frequencies, interconnected nonlinear relationship (Table 1). In a fractured carbonate reservoir, after acoustic impact,

Table 1. Discrete frequencies from the spectrum of the acoustic emission signal at a depth of 2840 meters.

\begin{tabular}{cccc}
\hline $\mathbf{N}$ & $\omega(N)$ & $\omega(\Sigma)$ & $\Delta \omega$ \\
\hline 1 & 1259 & & \\
2 & 2648 & & \\
3 & 4048 & $\omega_{1}+\omega_{2}$ & $-3.50 \%$ \\
4 & 6007 & & \\
5 & 6826 & $\omega_{2}+\omega_{3}$ & $-1.90 \%$ \\
6 & 8290 & $\omega_{2}+\omega_{4}$ & $4.40 \%$ \\
7 & 9506 & $\omega_{1}+\omega_{6}$ & $-0.45 \%$ \\
8 & 10,389 & $\omega_{3}+\omega_{4}$ & $3.21 \%$ \\
9 & 11,509 & $\omega_{3}+\omega_{5}$ & $5.52 \%$ \\
\hline
\end{tabular}




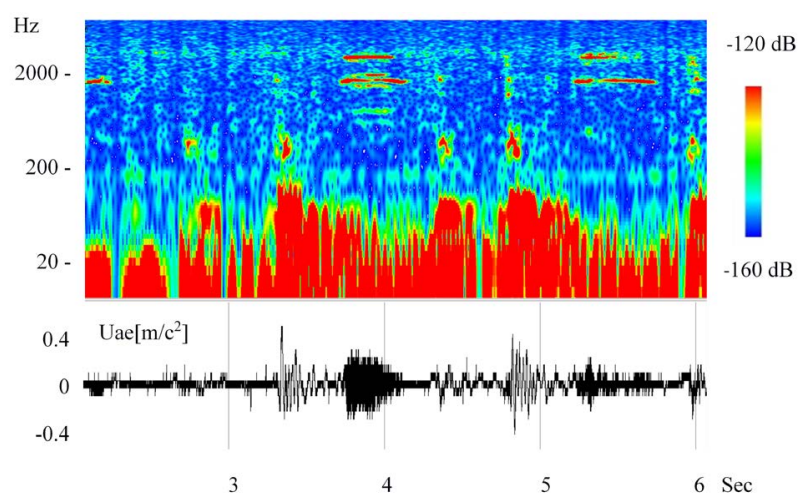

Figure 1. Acoustic emission signal and its spectrogram after acoustic impact in an oil well at a depth of 2840 meters in a terrigenous-type reservoir. $U$ is the amplitude of acoustic responce, axes $\mathrm{X}$ is time in sec.

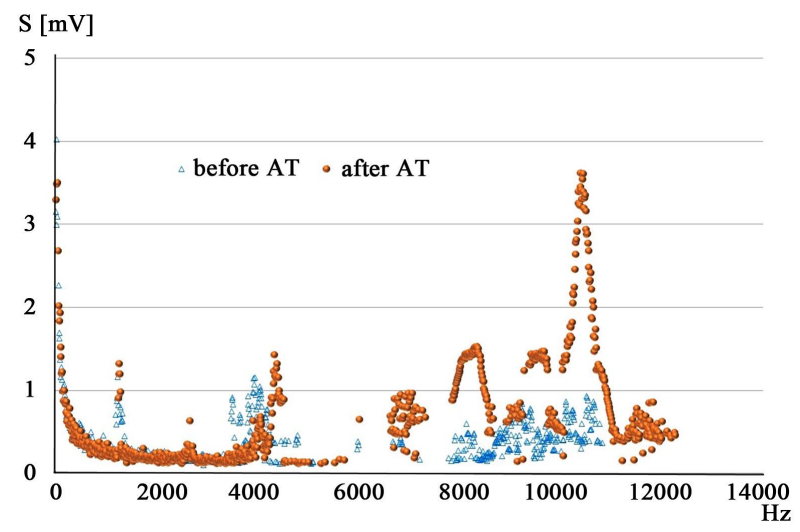

Figure 2. The spectrum of the acoustic emission signal at the point of $2840 \mathrm{~m}$ of the terrigenous collector before (AT, blue) and after (AT, bown) the acoustic impact. $\mathrm{S}$ is the spectrum amplitude.

the AE signal also has a pronounced discrete character. Moreover, after the acoustic impact, there was a significant increase in the energy of the induced acoustic emission compared with the background and the formation of pronounced discrete frequencies of energy in the spectrum of the emission signal. The nonlinearity of the radiation process caused by acoustic emission is manifested in the ratio of the main frequencies of the spectrum. But at the same time, there is a significant difference in the composition of discrete frequencies of the terrigenous and carbonate type spectra. The fractured carbonate type of collector has a much smaller number of discrete frequencies and their location in a narrower band as compared with the emission spectrum of a terrigenous collector. This is due to the different structure of the pore space of these reservoirs and the nature of changes in the dynamic processes of the stress state in them and the filtration of fluids.

In the column $\omega(N)$ it is the frequency from the spectrum in Figure 2, in the column $\omega(\Sigma)$ is the sum of the frequencies of the various vibration modes, and in the column $\Delta \omega$ is the difference of the frequency values measured and calculated. 
The main result of this part of our research consists on that fact that the resonant frequencies of the acoustic response and their sums are in a good agreement with the measured ones: so $\omega_{1}+\omega_{2}=\omega_{3}:(1259+2648)=3907$ and the measured frequency $\omega_{3}$ is 4048 , then the difference between the measured and calculated value of $\omega_{3}$ is $-3.5 \%$, that value is less than the error of experimental data and that coincides with the result of connection two oscillations in a nonlinear medium [1].

\section{Sound Diffraction Simulation on a Two-Dimensional Porous Oil-Saturated Reservoir of a Hierarchical Type, Located in an N-Layer Elastic Medium after Acoustic Excitation}

Here we shall construct an algorithm for modeling the resonant acoustic response process of a two-phase porous hierarchical medium according to the results written in Table 1 . We shall assume that the number $\mathrm{N}$ in the table will coincide with the number of the rank of hierarchical inclusions in the elastic reservoir. These inclusions will be of different features: the may have elastic features, plastic features and can be oil saturated. They can be a large inclusion, that consists of some number of fewer inclusions. And these features are linked with the sequence of 1 up to 9 of $\mathrm{N}$ in Table 1 .

In the paper [5] algorithm for modeling sound diffraction on 2D elastic hierarchical inclusion located in J-th layer of the N-layered medium is written using such designations and functions.

$G_{S p, j}\left(M, M^{0}\right)$-source function of seismic field, for which the boundary problem had been formulated in the paper [6]; $k_{1 j i}^{2}=\omega^{2}\left(\sigma_{j i} / \lambda_{j i}\right)$-wave number for the longitudinal wave; the index $j i$ denotes the identity of the properties of the medium within the heterogeneity, ja-out of heterogeneity; $\lambda$-constant Lame; $\sigma$-density of the medium; $\omega$-circular frequency; $\boldsymbol{u}=\operatorname{grad} \varphi$ -displacement vector; $\varphi^{0}$-potential of a normal seismic field in a layered medium in the absence of heterogeneity: $\varphi_{j i}^{0}=\varphi_{j a}^{0} \cdot l=1, \cdots, L$-rank hierarchy of inclusions. Let $l=1 . \lambda_{j i}=\lambda_{j a}+\omega_{1} \lambda_{j i(l=1)}[6] . \varphi_{(l=1)-1}^{0}$ corresponds to the exciting field at the reservoir level. The system of equations [5] will be rewritten as (1):

$$
\begin{aligned}
& \frac{\left(k_{1 j i(l=1)}^{2}-k_{1 j}^{2}\right)}{2 \pi} \iint_{S c(l=1)} \varphi_{(l=1)}(M) G_{S p, j}\left(M, M^{0}\right) \mathrm{d} \tau_{M}+\frac{\sigma_{j a}}{\sigma_{j i}} \varphi_{(l=1)-1}^{0}\left(M^{0}\right) \\
& -\frac{\left(\sigma_{j a}-\sigma_{j i(l=1)}\right)}{\sigma_{j i(l=1)} 2 \pi} \oint_{C(l=1)} G_{S p, j} \frac{\partial \varphi_{(l=1)}}{\partial n} \mathrm{~d} c=\varphi_{(l=1)}\left(M^{0}\right), M^{0} \in S_{C(l=1)}, \\
& \frac{\sigma_{j i(l=1)}\left(k_{1 j i(l=1)}^{2}-k_{1 j}^{2}\right)}{\sigma\left(M^{0}\right) 2 \pi} \iint_{S c(l=1)} \varphi_{(l=1)}(M) G_{S p, j}\left(M, M^{0}\right) \mathrm{d} \tau_{M}+\varphi_{(l=1)-1}^{0}\left(M^{0}\right) \\
& -\frac{\left(\sigma_{j a}-\sigma_{j i(l=1)}\right)}{\sigma\left(M^{0}\right) 2 \pi} \oint_{C(l=1)} G_{S p, j} \frac{\partial \varphi_{(l=1)}}{\partial n} \mathrm{~d} c=\varphi_{(l=1)}\left(M^{0}\right), M^{0} \notin S_{C(l=1)}
\end{aligned}
$$


$I=I+1$. that is: $I=2$, inclusions $S c_{(l=2)}$ and $C_{(l=2)}$ are located inside sections $S c_{(l=1)}$ and contours $C_{(l=1)}$ correspondingly, $\lambda_{j i}=\lambda_{j i(l=1)}+\omega_{2} \lambda_{j i(l=2)}$, the inclusions are plastic, $\omega_{2}$ is taken from Table $1 . \varphi_{(l=2)-1}^{0}=\varphi_{(l=1)}$. The system of Equations (1) is rewritten as:

$$
\begin{aligned}
& \frac{\left(k_{1 j i(l=2)}^{2}-k_{1 j}^{2}\right)}{2 \pi} \iint_{S c(l=2)} \varphi_{(l=2)}(M) G_{S p, j}\left(M, M^{0}\right) \mathrm{d} \tau_{M}+\frac{\sigma_{j a}}{\sigma_{j i(l=2)}} \varphi_{(l=2)-1}^{0}\left(M^{0}\right) \\
& -\frac{\left(\sigma_{j a}-\sigma_{j i(l=2)}\right)}{\sigma_{j i(l=2)} 2 \pi} \oint_{C(l=2)} G_{S p, j} \frac{\partial \varphi_{(l=2)}}{\partial n} \mathrm{~d} c=\varphi_{(l=2)}\left(M^{0}\right), M^{0} \in S_{C(l=2)}, \\
& \frac{\sigma_{j i(l=2)}\left(k_{1 j i(l=2)}^{2}-k_{1 j}^{2}\right)}{\sigma\left(M^{0}\right) 2 \pi} \iint_{S c(l=2)} \varphi_{(l=2)}(M) G_{S p, j}\left(M, M^{0}\right) \mathrm{d} \tau_{M}+\varphi_{(l=2)-1}^{0}\left(M^{0}\right) \\
& -\frac{\left(\sigma_{j a}-\sigma_{j i(l=2)}\right)}{\sigma\left(M^{0}\right) 2 \pi} \oint_{C(l=2)} G_{S p, j} \frac{\partial \varphi_{(l=2)}}{\partial n} \mathrm{~d} c=\varphi_{(l=2)}\left(M^{0}\right), M^{0} \notin S_{C(l=2)} .
\end{aligned}
$$

$I=1+1$. that is: $I=3$, inclusions $S c_{(l=3) m}$ and $C_{(l=3) m}$ are located inside $S c_{(l=2)}$ and $C_{(l=2)}, m=1, \cdots, M$ correspondingly, $\lambda_{j i}=\lambda_{j i(l=2)}+\omega_{3} \lambda_{j i(l=3)}$, the inclusions are plastic also, $\omega_{3}$ is taken from Table 1. $\varphi_{(l=3)-1}^{0}=\varphi_{(l=2)}$.

The system of Equations (1) will be rewritten as [7]:

$$
\begin{aligned}
& \sum_{1}^{M}\left\{\frac{\left(k_{1 j i(l=3) m}^{2}-k_{1 j}^{2}\right)}{2 \pi} \int_{S c(l=3) m} \varphi_{(l=3) m}(M) G_{S p, j}\left(M, M^{0}\right) \mathrm{d} \tau_{M}+\frac{\sigma_{j a}}{\sigma_{j i(l=3) m}} \varphi_{(l=3)-1}^{0}\left(M^{0}\right)\right. \\
& \left.-\frac{\left(\sigma_{j a}-\sigma_{j i(l=3) m}\right)}{\sigma_{j i(l=3) m} 2 \pi} \oint_{C(l=3) m} G_{S p, j} \frac{\partial \varphi_{(l=3) m}}{\partial n} \mathrm{~d} c=\varphi_{(l=3) m}\left(M^{0}\right)+\left(\alpha p_{2}\right)_{m}\right\}, M^{0} \in S_{C(l=3) m}, \\
& \frac{\sigma_{j i(l=3)}\left(k_{1 j i(l=3)}^{2}-k_{1 j}^{2}\right)}{\sigma\left(M^{0}\right) 2 \pi} \int_{S c(l=3)} \varphi_{(l=3)}(M) G_{S p, j}\left(M, M^{0}\right) \mathrm{d} \tau_{M}+\varphi_{(l=3)-1}^{0}\left(M^{0}\right) \\
& -\frac{\left(\sigma_{j a}-\sigma_{j i(l=3)}\right)}{\sigma\left(M^{0}\right) 2 \pi} \oint_{C(l=3)} G_{S p, j} \frac{\partial \varphi_{(l=3)} \mathrm{d} c=\varphi_{(l=3)}\left(M^{0}\right), M^{0} \notin S_{C(l=3) m} m=1, \cdots, M .}{\partial n} m \\
& \left(\alpha p_{2}\right)_{m}=\left(\left(1-\chi-\frac{K}{K_{0}}\right) p_{2}\right)_{m}
\end{aligned}
$$

$K$-compression module, $\chi$-porosity, $K_{0}$-true module of phase compressibility, $p_{2}$-pore hydrostatic pressure for the $m$-th fluid-saturated inclusion and $\omega_{3}=\omega_{1}+\omega_{2}$; (these frequency values are taken from Table 1). As we can see there are some inclusions which contain the fluid. So for each inclusion the features of $\left(\alpha p_{2}\right)$ for each $m$ are different and they are located inside the inclusions sections $S c_{(l=2)}$ and contours $C_{(l=2)}$.

$l=I+1$, that is $l=4$, inclusions $S c_{(l=4)}$ and $C_{(l=4)}$ are located inside sections $S c_{(l=2)}$ and contours $C_{(l=2)}$ correspondingly, $\lambda_{j i}=\lambda_{j i(l=2)}+\omega_{4} \lambda_{j i(l=4)}$, $\omega_{4}$ is taken from Table 1. $\varphi_{(l=4)-1}^{0}=\varphi_{(l=2)}$. The system of equations [5] will be rewritten as: 


$$
\begin{aligned}
& \frac{\left(k_{1 j i(l=4)}^{2}-k_{1 j}^{2}\right)}{2 \pi} \iint_{S c(l=4)} \varphi_{(l=4)}(M) G_{S p, j}\left(M, M^{0}\right) \mathrm{d} \tau_{M}+\frac{\sigma_{j a}}{\sigma_{j i(l=4)}} \varphi_{(l=4)-1}^{0}\left(M^{0}\right) \\
& -\frac{\left(\sigma_{j a}-\sigma_{j i(l=4)}\right)}{\sigma_{j i(l=4)} 2 \pi} \oint_{C(l=4)} G_{S p, j} \frac{\partial \varphi_{(l=4)}}{\partial n} \mathrm{~d} c=\varphi_{(l=4)}\left(M^{0}\right), M^{0} \in S_{C(l=4)}, \\
& \frac{\sigma_{j i(l=4)}\left(k_{1 j i(l=4)}^{2}-k_{1 j}^{2}\right)}{\sigma\left(M^{0}\right) 2 \pi} \iint_{S c(l=4)} \varphi_{(l=4)}(M) G_{S p, j}\left(M, M^{0}\right) \mathrm{d} \tau_{M}+\varphi_{(l=4)-1}^{0}\left(M^{0}\right) \\
& -\frac{\left(\sigma_{j a}-\sigma_{j i(l=4)}\right)}{\sigma\left(M^{0}\right) 2 \pi} \oint_{C(l=4)} G_{S p, j} \frac{\partial \varphi_{(l=4)}}{\partial n} \mathrm{~d} c=\varphi_{(l=4)}\left(M^{0}\right), M^{0} \notin S_{C(l=4)} .
\end{aligned}
$$

$I=1+1$, that is $I=5$, inclusions $S c_{(l=5) m m}$ and $C_{(l=5) m m}$ are located inside the sections $S c_{(l=2)}$ and contours $C_{(l=2)}, \quad m m=1, \cdots, M M$ correspondingly, $\lambda_{j i}=\lambda_{j i(l=4)}+\omega_{5} \lambda_{j i(l=5)}, \quad \varphi_{(l=5)-1}^{0}=\varphi_{(l=2)}$. The system of equations [5] will be rewritten as [7]:

$$
\begin{aligned}
& \sum_{1}^{M M}\left\{\frac{\left(k_{1 j i(l=5) m m}^{2}-k_{1 j}^{2}\right)}{2 \pi} \int_{S c(l=5) m m} \varphi_{(l=5) m m}(M) G_{S p, j}\left(M, M^{0}\right) \mathrm{d} \tau_{M}+\frac{\sigma_{j a}}{\sigma_{j i(l=5) m m}} \varphi_{(l=5)-1}^{0}\left(M^{0}\right)\right. \\
& \left.-\frac{\left(\sigma_{j a}-\sigma_{j i(l=5) m m}\right)}{\sigma_{j i(l l=5) m m} 2 \pi} \oint_{C(l=5) m m} G_{S p, j} \frac{\partial \varphi_{(l=5) m m}}{\partial n} \mathrm{~d} c=\varphi_{(l=5) m m}\left(M^{0}\right)+\left(\alpha p_{2}\right)_{m m}\right\}, M^{0} \in S_{C(l=5) m m}, \\
& \frac{\sigma_{j i(l=5)}\left(k_{1 j i(l=5)}^{2}-k_{1 j}^{2}\right)}{\sigma\left(M^{0}\right) 2 \pi} \iint_{S c(l=5)} \varphi_{(l=5)}(M) G_{S p, j}\left(M, M^{0}\right) \mathrm{d} \tau_{M}+\varphi_{(l=5)-1}^{0}\left(M^{0}\right) \\
& -\frac{\left(\sigma_{j a}-\sigma_{j i(l=5)}\right)}{\sigma\left(M^{0}\right) 2 \pi} \oint_{C(l=5)} G_{S p, j} \frac{\partial \varphi_{(l=5)}}{\partial n} \mathrm{~d} c=\varphi_{(l=5)}\left(M^{0}\right), M^{0} \notin S_{C(l=5) m m} m m=1, \cdots, M M . \\
& \left(\alpha p_{2}\right)_{m m}=\left(\left(1-\chi-\frac{K}{K_{0}}\right) p_{2}\right)_{m m} ; \omega_{5}=\omega_{2}+\omega_{3} ; \quad \text { (these frequency values are }
\end{aligned}
$$
taken from Table 1).

$l=1+1$, that is $l=6$, inclusions $S c_{(l=6) m n}$ and $C_{(l=6) m n}$ are located inside the sections $S c_{(l=2)}$ and contours $C_{(l=2)}, \quad m n=1, \cdots, M N$ correspondingly, $\lambda_{j i}=\lambda_{j i(l=2)}+\omega_{6} \lambda_{j i(l=6)}, \quad \varphi_{(l=6)-1}^{0}=\varphi_{(l=2)}$. The system of equations [5] will be rewritten as [7]:

$$
\begin{aligned}
& \sum_{1}^{M N}\left\{\frac{\left(k_{1 j i(l=6) m n}^{2}-k_{1 j}^{2}\right)}{2 \pi} \int_{S c(l=6) m n} \varphi_{(l=6) m n}(M) G_{S p, j}\left(M, M^{0}\right) \mathrm{d} \tau_{M}+\frac{\sigma_{j a}}{\sigma_{j i(l=6) m n}} \varphi_{(l=6)-1}^{0}\left(M^{0}\right)\right. \\
& \left.-\frac{\left(\sigma_{j a}-\sigma_{j i(l=6) m n}\right)}{\sigma_{j i(l=6) m n} 2 \pi} \oint_{C(l=6) m n} G_{S p, j} \frac{\partial \varphi_{(l=6) m n}}{\partial n} \mathrm{~d} c=\varphi_{(l=6) m n}\left(M^{0}\right)+\left(\alpha p_{2}\right)_{m n}\right\}, M^{0} \in S_{C(l=6) m n}, \\
& \frac{\sigma_{j i(l=6)}\left(k_{1 j i(l=6)}^{2}-k_{1 j}^{2}\right)}{\sigma\left(M^{0}\right) 2 \pi} \iint_{S c(l=6)} \varphi_{(l=6)}(M) G_{S p, j}\left(M, M^{0}\right) \mathrm{d} \tau_{M}+\varphi_{(l=6)-1}^{0}\left(M^{0}\right) \\
& -\frac{\left(\sigma_{j a}-\sigma_{j i(l=6)}\right)}{\sigma\left(M^{0}\right) 2 \pi} \oint_{C(l=6)} G_{S p, j} \frac{\partial \varphi_{(l=6)}}{\partial n} \mathrm{~d} c=\varphi_{(l=6)}\left(M^{0}\right), M^{0} \notin S_{C(l=6) m n} m n=1, \cdots, M N .
\end{aligned}
$$


$\left(\alpha p_{2}\right)_{m n}=\left(\left(1-\chi-\frac{K}{K_{0}}\right) p_{2}\right)_{m n} ; \omega_{6}=\omega_{2}+\omega_{4} ;$ (these frequency values are taken from Table 1).

$l=1+1$. that is $l=7$, inclusions $S c_{(l=7) n m}$ и $C_{(l=7) n m}$ are located inside the section $S c_{(l=1)}$ and contour $C_{(l=1)}, \quad n m=1, \cdots, N M \quad$ correspondingly, $\lambda_{j i}=\lambda_{j i(l=1)}+\omega_{7} \lambda_{j i(l=7)}, \quad \varphi_{(l=7)-1}^{0}=\varphi_{(l=1)}$. The system of Equations (1) will be rewritten as [8]:

$\sum_{1}^{N M}\left\{\frac{\left(k_{1 j i(l=7) n m}^{2}-k_{1 j}^{2}\right)}{2 \pi} \iint_{S c(l=7) n m} \varphi_{(l=7) n m}(M) G_{S p, j}\left(M, M^{0}\right) \mathrm{d} \tau_{M}+\frac{\sigma_{j a}}{\sigma_{j i(l=7) n m}} \varphi_{(l=7)-1}^{0}\left(M^{0}\right)\right.$ $\left.-\frac{\left(\sigma_{j a}-\sigma_{j i(l=7) n m}\right)}{\sigma_{j i(l=7) n m} 2 \pi} \oint_{C(l=7) n m} G_{S p, j} \frac{\partial \varphi_{(l=7) n m}}{\partial n} \mathrm{~d} c=\varphi_{(l=7) n m}\left(M^{0}\right)+\left(\alpha p_{2}\right)_{n m}\right\}, M^{0} \in S_{C(l=7) n m}$,

$\frac{\sigma_{j i(l=7)}\left(k_{1 j i(l=7)}^{2}-k_{1 j}^{2}\right)}{\sigma\left(M^{0}\right) 2 \pi} \iint_{S c(l=7)} \varphi_{(l=7)}(M) G_{S p, j}\left(M, M^{0}\right) \mathrm{d} \tau_{M}+\varphi_{(l=7)-1}^{0}\left(M^{0}\right)$

$-\frac{\left(\sigma_{j a}-\sigma_{j i(l=7)}\right)}{\sigma\left(M^{0}\right) 2 \pi} \oint_{C(l=7)} G_{S p, j} \frac{\partial \varphi_{(l=7)}}{\partial n} \mathrm{~d} c=\varphi_{(l=7)}\left(M^{0}\right), M^{0} \notin S_{C(l=7) n m} n m=1, \cdots, N M$.

$\left(\alpha p_{2}\right)_{n m}=\left(\left(1-\chi-\frac{K}{K_{0}}\right) p_{2}\right)_{n m} ; \omega_{7}=\omega_{1}+\omega_{6} ; \quad$ (these frequency values are taken from Table 1).

$I=I+1$, that is $I=8$, inclusions with sections $S c_{(l=8) m}$, and contours $C_{(l=8) m}$, are located inside the section $S c_{(l=4)}$ and contour $C_{(l=4)}, m^{\prime}=1, \cdots, M^{\prime}$ correspondingly, $\lambda_{j i}=\lambda_{j i(l=4)}+\omega_{8} \lambda_{j i(l=8) m^{\prime}}, \quad \varphi_{(l=8)-1}^{0}=\varphi_{(l=4)}$. The system of equations [5] will be rewritten as [7]:

$\sum_{1}^{M^{\prime}}\left\{\frac{\left(k_{1 j i(l=8) m^{\prime}}^{2}-k_{1 j}^{2}\right)}{2 \pi} \iint_{S c(l=8) m^{\prime}} \varphi_{(l=8) m^{\prime}}(M) G_{S p, j}\left(M, M^{0}\right) \mathrm{d} \tau_{M}+\frac{\sigma_{j a}}{\sigma_{j i(l=8) m^{\prime}}} \varphi_{(l=8)-1}^{0}\left(M^{0}\right)\right.$

$\left.-\frac{\left(\sigma_{j a}-\sigma_{j i(l=8) m^{\prime}}\right)}{\sigma_{j i(l=8) m^{\prime}} 2 \pi} \oint_{C(l=8) m^{\prime}} G_{S p, j} \frac{\partial \varphi_{(l=8) m^{\prime}}}{\partial n} \mathrm{~d} c=\varphi_{(l=8) m^{\prime}}\left(M^{0}\right)+\left(\alpha p_{2}\right)_{m}\right\}, M^{0} \in S_{C(l=8) m^{\prime}}$,

$\frac{\sigma_{j i(l=8)}\left(k_{1 j i(l=8)}^{2}-k_{1 j}^{2}\right)}{\sigma\left(M^{0}\right) 2 \pi} \iint_{S c(l=8)} \varphi_{(l=8)}(M) G_{S p, j}\left(M, M^{0}\right) \mathrm{d} \tau_{M}+\varphi_{(l=8)-1}^{0}\left(M^{0}\right)$

$$
-\frac{\left(\sigma_{j a}-\sigma_{j i(l=8)}\right)}{\sigma\left(M^{0}\right) 2 \pi} \oint_{C(l=8)} G_{S p, j} \frac{\partial \varphi_{(l=8)}}{\partial n} \mathrm{~d} c=\varphi_{(l=8)}\left(M^{0}\right), M^{0} \notin S_{C(l=8) m^{\prime}} m^{\prime}=1, \cdots, M^{\prime} .
$$

$\left(\alpha p_{2}\right)_{m^{\prime}}=\left(\left(1-\chi-\frac{K}{K_{0}}\right) p_{2}\right)_{m^{\prime}} ; \omega_{8}=\omega_{4}+\omega_{3} ; \quad$ (these frequency values are taken from Table 1).

$I=I+1$, that is $I=9$, inclusions with sections $S c_{(l=9) m m m}$ and contours $C_{(l=9) \mathrm{mmm}}$ are located inside the sections $S c_{(l=3)}$ and $S c_{(l=5)}$ and contours $C_{(l=3)}$, and $C_{(l=5)} \quad m=1, \cdots, M$ and $m m=1, \cdots, M M$ correspondingly, 


$$
\begin{aligned}
& \sum_{1}^{M M M}\left\{\frac{\left(k_{1 j i(l=9) m m}^{2}-k_{1 j}^{2}\right)}{2 \pi} \int_{S c(l=9) m m m} \varphi_{(l=9) m m m}(M) G_{S p, j}\left(M, M^{0}\right) \mathrm{d} \tau_{M}+\frac{\sigma_{j a}}{\sigma_{j i(l=9) m m m}} \varphi_{(l=9)-1}^{0}\left(M^{0}\right)\right. \\
& \left.-\frac{\left(\sigma_{j a}-\sigma_{j i(l=9) m m m}\right)}{\sigma_{j i(l=9) m m m} 2 \pi} \oint_{C(l=9) m m m} G_{S p, j} \frac{\partial \varphi_{(l=9) m m m}}{\partial n} \mathrm{~d} c=\varphi_{(l=9) m m m}\left(M^{0}\right)+\left(\alpha p_{2}\right)_{m m m}\right\}, M^{0} \in S_{C(l=5) m m m}, \\
& \frac{\sigma_{j i(l=9)}\left(k_{1 j i(l=9)}^{2}-k_{1 j}^{2}\right)}{\sigma\left(M^{0}\right) 2 \pi} \int_{S c(l=9)} \varphi_{(l=9)}(M) G_{S p, j}\left(M, M^{0}\right) \mathrm{d} \tau_{M}+\varphi_{(l=9)-1}^{0}\left(M^{0}\right) \\
& -\frac{\left(\sigma_{j a}-\sigma_{j i(l=9)}\right)}{\sigma\left(M^{0}\right) 2 \pi} \oint_{C(l=9)} G_{S p, j} \frac{\partial \varphi_{(l=9)}}{\partial n} \mathrm{~d} c=\varphi_{(l=9)}\left(M^{0}\right), M^{0} \notin S_{C(l=9) m m m} m m m=1, \cdots, M M M \\
& \left(\alpha p_{2}\right)_{m m m}=\left(\left(1-\chi-\frac{K}{K_{0}}\right) p_{2}\right)_{m m m} ; \omega_{9}=\omega_{5}+\omega_{3} ; \text { (these frequency values are }
\end{aligned}
$$
taken from Table 1$)$.

\section{Conclusions}

Thus, acoustic emission in porous media of reservoir rocks has the ability of permanently acting radiation. The main feature of acoustic emission is a reflection of stress relaxation and high sensitivity to external influences. In the case of fluid-filled pore space, the medium generates an induced energy of elastic energy that is adequately associated with its properties and state, and the radiation is non-linear.

Using data from Table 1, a variant of the system of structural adjustment algorithms for a nonlinear two-phase porous medium after its dynamic excitation is constructed. The values of the resonant frequencies are taken from the table. The rank of fluid saturated inclusions located in a porous medium with plastic properties is correlated with the number of the resonant frequencies. Such a system of algorithms was developed for the first time. That system can vary by the features (elastic, plastic and saturated) of the inclusions. By that analysis, we can take into account how many saturated inclusions can move inside the reservoir and can increase the potential oil recovery. After the first acoustic impact if it is needed, we can repeat the second impact, formulate the analogue of Table 1 and construct the system of algorithms linked with data of a new table.

\section{Conflicts of Interest}

The authors declare no conflicts of interest regarding the publication of this paper.

\section{References}

[1] Rabinovich, M. and Trubetskov, D. (2000) Introduction to the Theory of Oscillations and Waves of SIC "Regular and Chaotic Dynamics" Moscow.

[2] Dryagin, V.V. (2018) The Use of Induced Acoustic Emission from Reservoirs for the Detection and Extraction of Hydrocarbons. Georesources, 20, 246-260. 
https://doi.org/10.18599/grs.2018.3.246-260

[3] Chebotareva, I.Y., Volodin, I.A. and Dryagin, V.V. (2016) Generation of a Low-Frequency Branch of Acoustic Emission in Affected Rocks. Doklady Earth Sciences, 468, 205-208. https://doi.org/10.1134/S1028334X16050160

[4] Chebotareva, I.Y., Volodin, I.A. and Dryagin, V.V. (2017) Acoustic Effects in the Deformation of Structually İnhomogeneous Media. Acoustical Physics, 63, 86-94. https://doi.org/10.1134/S106377101606004X

[5] Hachay, O.A. and Khachay, A.Y. (2013) Modeling of Seismic and Electromagnetic Fields in Hierarchically Heterogeneous Environments. In: Bulletin of the South Ural State University. Series "Computational Mathematics and Computer Science", 2, 48-56. https://doi.org/10.14529/cmse130204

[6] Hachay, O. and Khachay, A. (2017) Acoustic Wave Monitoring of Fluid Dynamic in the Rock Massif with Anomaly Density, Stressed and Plastic Hierarchic Inclusion. Computational and Experimental Studies of Acoustic Waves, Intec Open, 2017, Chapter 4, pp. 63-80. https://doi.org/10.5772/intechopen.70590

[7] Hachay, O. and Khachay, A. (2014) Reflection of the Processes of Non-Equilibrium Two-Phase Filtration in Oil-Saturated Hierarchical Media in the Data of Active Wave Geophysical Monitoring. GIAB, 4, 232-238.

[8] Hachay, O. and Khachay, A. (2016) Modeling the Propagation of a Seismic Field in a Layered-Block Elastic Medium with Hierarchical Plastic İnclusions. GIAB, 12, 318-326. 